Mon. Not. R. astr. Soc. (1985) 215, 713-730

\title{
Best approximate quadratic integrals in stellar dynamics
}

\author{
P. T. de Zeeuw` ${ }^{\star}$ Sterrewacht Leiden, Postbus 9513,2300 RA Leiden, The \\ Netherlands \\ D. Lynden-Bell Institute of Astronomy, Madingley Road, Cambridge СВ3 ОНА
}

Accepted 1985 March 4. Received 1985 March 4; in original form 1984 June 25

Summary. The nature of the integrals of motion in Stäckel potentials, for which the equations of motion separate in ellipsoidal coordinates, is elucidated. The problem of fitting a general potential with one of Stäckel form is considered, both locally and globally.

In local fitting the potential is expanded around an equilibrium point and this expansion is compared term by term with the similar expansion of a Stäckel potential. This is done explicitly for potentials with three reflection symmetries. Expansions are given for the integrals of motion in the best-fitting Stäckel potential. They may be expressed directly in terms of the expansion coefficients of the potential that is fitted.

The results of local fitting are applied to the gravitational potentials of ellipsoidal density distributions, and also to ellipsoidal potentials. It is shown that there is a unique inhomogeneous density distribution stratified on similar ellipsoids of arbitrary axis ratios with a potential that is exactly of Stäckel form.

A method is presented for the global fitting of a triaxial potential with one of Stäckel form. The foci of the ellipsoidal coordinates play an important role in this procedure.

\section{Introduction}

The phase-flow in many smooth potentials possesses one or more approximate integrals besides the energy (Contopoulos 1960), and it has become important to obtain simple approximations for them.

In stellar dynamics the special potentials in which the motion is separable, or for which other integrals exist, have been to some extent classified (Eddington 1915; Clark 1936; Lynden-Bell 1962). For more general potentials, Whittaker (1917) in his book Analytical Dynamics already gives formal series for extra integrals, but no criteria for their convergence. He calls these integrals adelphic integrals, since their properties resemble closely those of the energy integral. Since then, many methods have been used for the formal construction of such integrals in a

${ }^{\star}$ Present address: Institute for Advanced Study, Princeton, NJ 08540, USA. 
variety of dynamical problems (e.g. Contopoulos 1963; Hagihara 1974). However, there remain many unsolved problems in the area.

Here we consider a specific problem. Knowing the forms of the exactly separable potentials we ask how closely can a given potential be fitted with a separable one.

The separable potentials were first used in stellar dynamics by Eddington (1915), but their history goes back much further (Jacobi 1866; Liouville 1846, 1849; Bertrand 1852; Stäckel 1890, 1891, 1893; Levi-Civita 1904; Dall'Acqua 1908). The general case is separable in ellipsoidal coordinates $(\lambda, \mu, v)$. They are the roots for $\tau$ of

$\frac{x^{2}}{\tau+\alpha}+\frac{y^{2}}{\tau+\beta}+\frac{z^{2}}{\tau+\gamma}=1$,

where $\alpha, \beta$ and $\gamma$ are three fixed numbers, and $-\gamma \leq v \leq-\beta \leq \mu \leq-\alpha \leq \lambda$. In these coordinates the equations of motion separate for all potentials $V_{\mathrm{S}}$ of the form (Weinacht 1924)

$V_{\mathrm{S}}=-\frac{\zeta^{*}(\lambda)}{(\lambda-\mu)(\lambda-v)}-\frac{\eta^{*}(\mu)}{(\mu-v)(\mu-\lambda)}-\frac{\theta^{*}(v)}{(v-\lambda)(v-\mu)}$,

where $\zeta^{*}(\lambda), \eta^{*}(\mu)$ and $\theta^{*}(v)$ are arbitrary functions. All other cases found by Stäckel can be derived from equations (1) and (2) by taking appropriate limits (see, e.g. Morse \& Feshbach 1953). All orbits in these Stäckel potentials have three explicit analytic integrals, and indeed all orbits in them can be determined in terms of simple quadratures.

Motion in the neighbourhood of an equilibrium point of a given potential $V$ is usually described as perturbed harmonic motion. One expands $V$ in a Taylor series in the displacements and in zeroth order retains only the quadratic terms. Thus, the unperturbed motion is harmonic. The effects of the higher order terms can then be calculated by means of standard perturbation methods. A technique that has been applied many times, and with considerable success, is the method of averaging (e.g. Bogoliubov \& Mitropolsky 1961; Verhulst 1979). In all cases this method provides one integral in addition to the energy integral. Whether in a three-dimensional potential a third integral exists has to be discovered from the averaged equations of motion however. This is in general quite a difficult task (Verhulst 1983). A major advantage of the averaging method is that it is able to treat resonances, and provide rigorous estimates of the accuracy of the results obtained. Furthermore, the method is very general; it readily extends to rotating potentials (de Zeeuw \& Merritt 1983).

van de Hulst (1962) showed that for potentials $V=V\left(x, y^{2}\right)$ it is often profitable to describe motion near an equilibrium point not as perturbed harmonic motion, as done, e.g. by Contopoulos (1960), but instead as (unperturbed) motion in a Stäckel potential. He found that by a proper choice of the coordinate system one can fit the expansion of $V$ up to and including quartic terms. As a result he obtained very accurate approximations to numerically calculated orbits.

Hori (1962) studied the global problem of fitting a general potential - in his case the Schmidt potential for the Galaxy - with a separable one (see also Wayman 1959). Hori proceeded to derive properties of stellar orbits that coincide with those that were deduced independently by Ollongren (1962), based on numerical results. Kuzmin (1956) had already proven some remarkable and useful theorems on the density corresponding to axisymmetric separable potentials, and had used one of these potentials for the Galaxy. Recently, Manabe (1979) tried several different procedures for fitting a separable potential to a model for the Galaxy.

Many elliptical galaxies may have triaxial figures, instead of axisymmetric ones (Binney 1978; Illingworth 1981). Stellar orbits in the corresponding triaxial potentials in many cases appear to possess two integrals of motion in addition to the energy integral (Schwarzschild 1979, 1981). Therefore, it is natural to ask how well a general potential can be fitted by a potential of Stäckel 
form. In particular, if the fitting can be done accurately globally for the potentials that are relevant for elliptical galaxies, then perturbation theory applied to the best-fitting Stäckel potential - rather than to a simple quadratic potential - should give a very good description of the orbits in these systems. Based on the results for axisymmetric systems mentioned in the above, it is to be expected that in many cases already the unperturbed motion in the best-fitting Stäckel potential provides an accurate description of the motion.

Given a non-separable potential $V$ our task is then to find the 'closest' approximate potential of the form (2) by choice of $\alpha, \beta$ and $\gamma$ - this defines the coordinate system - and the functions $\zeta^{*}(\lambda)$, $\eta^{*}(\mu)$ and $\theta^{*}(v)$. We will consider the local fitting around a stable equilibrium point as well as the global fitting of a general potential.

Following Whittaker (1917), we shall refer to the extra integrals of motion that exist in Stäckel potentials as adelphic integrals, in order to distinguish them from the classical energy and angular momentum integrals. The adelphic integrals are quadratic in the velocities, as are the classical integrals. Thus, if the Stäckel potential $V_{\mathrm{S}}$ is - in a certain sense - the best fit to a potential $V$ then the integrals in $V_{\mathrm{S}}$ will be the best approximate quadratic integrals for motion in the potential $V$, in the same sense.

In the present paper we do not investigate the accuracy of the approximate integrals that we derive. Hence, our results are formal. The accuracy of local fitting can be assessed by means of a comparison with the averaging method. This we do in a companion paper (de Zeeuw 1985b).

In Section 2 we define our notation and discuss the adelphic integrals. Local fitting is considered in Section 3. Two useful applications are given in Section 4. We study global fitting in Section 5 .

\section{Stäckel potentials and their integrals of motion}

Ellipsoidal coordinates and Stäckel potentials have been discussed many times (e.g. Jacobi 1866; Whittaker \& Watson 1902; Morse \& Feshbach 1953). Here we summarize the properties that we shall need, and we show explicitly how the adelphic integrals are related to the angular momentum integrals that exist in spherical and axisymmetric potentials. Our notation follows that of Lynden-Bell (1962).

\subsection{NOTATION}

The ellipsoidal coordinates $(\lambda, \mu, v)$ are defined in equation (1). Expressions for $x, y$ and $z$ in terms of $\lambda, \mu$ and $v$ are

$x^{2}=\frac{(\lambda+\alpha)(\mu+\alpha)(v+\alpha)}{(\alpha-\beta)(\alpha-\gamma)}$, etc.

By 'etc.' we imply - here and below - similar relations with $\lambda \rightarrow \mu \rightarrow v \rightarrow \lambda, \alpha \rightarrow \beta \rightarrow \gamma \rightarrow \alpha$, $x \rightarrow y \rightarrow z \rightarrow x, P \rightarrow Q \rightarrow R \rightarrow P, \zeta \rightarrow \eta \rightarrow \theta \rightarrow \zeta, \ldots$

The coordinate surfaces are ellipsoids $(\lambda)$, hyperboloids of one sheet $(\mu)$, and hyperboloids of two sheets $(v)$. These surfaces are described by de Zeeuw (1985a), and are illustrated in Fig. 1. In each of the principal planes $x=0, y=0$ and $z=0$ the coordinates are elliptic coordinates (Fig. 2). The metric $d s^{2}$ is equal to $d x^{2}+d y^{2}+d z^{2}=P^{2} d \lambda^{2}+Q^{2} d \mu^{2}+R^{2} d v^{2}$ where

$P^{2}=\frac{(\lambda-\mu)(\lambda-v)}{4(\lambda+\alpha)(\lambda+\beta)(\lambda+\gamma)}$, etc.

The Hamiltonian $H$ for motion in a potential $V(\lambda, \mu, v)$ is

$H=\frac{p_{\lambda}^{2}}{2 P^{2}}+\frac{p_{\mu}^{2}}{2 Q^{2}}+\frac{p_{v}^{2}}{2 R^{2}}+V(\lambda, \mu, v)$, 


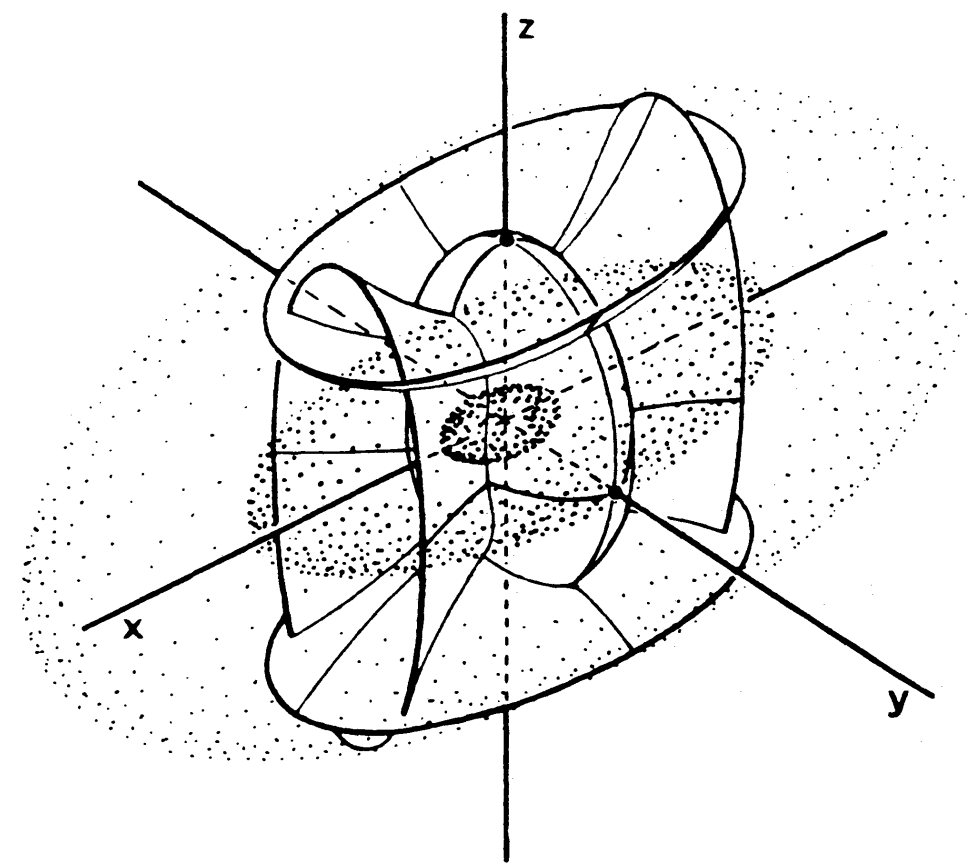

Figure 1. Ellipsoidal coordinates $(\lambda, \mu, v)$. The coordinate surfaces are ellipsoids and hyperboloids of one and two sheets. The density corresponding to a potential of Stäckel form in these coordinates is indicated.

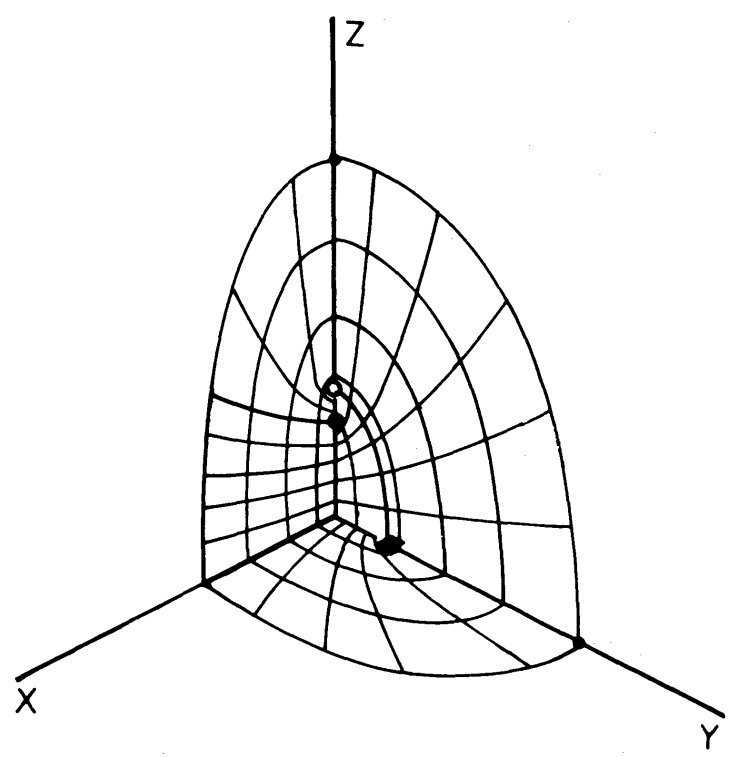

Figure 2. In each principal plane the ellipsoidal coordinates are elliptic coordinates.

where $p_{\lambda}=P^{2} \dot{\lambda}$, etc. are the momenta conjugate to $\lambda, \mu$ and $v$. The most general form of the potential $V_{\mathrm{S}}$ for which the equations of motion are separable in these coordinates is ( $\left.c f .2\right)$

$V_{\mathrm{S}}=-\frac{\zeta(\lambda)}{P^{2}}-\frac{\eta(\mu)}{Q^{2}}-\frac{\theta(v)}{R^{2}}=-\frac{\zeta^{*}(\lambda)}{(\lambda-\mu)(\lambda-v)}-\frac{\eta^{*}(\mu)}{(\mu-v)(\mu-\lambda)}-\frac{\theta^{*}(v)}{(v-\lambda)(v-\mu)}$,

where $\zeta^{*}(\lambda)=4(\lambda+\alpha)(\lambda+\beta)(\lambda+\gamma) \zeta(\lambda)$ and $\zeta(\lambda)$ is an arbitrary function of $\lambda$, etc. 


\subsection{INTEGRALS OF MOTION}

A Stäckel potential (6) admits three independent isolating integrals of motion that are quadratic in the velocities, the energy integral $H$ and two adelphic integrals $J$ and $K$ :

$H=X+Y+Z$,

$J=(\mu+v) X+(v+\lambda) Y+(\lambda+\mu) Z$,

$K=\mu v X+v \lambda Y+\lambda \mu Z$,

where $X=\left[1 / 2 p_{\lambda}^{2}-\zeta(\lambda)\right] / P^{2}$, etc.

From equation (3) it follows that

$\frac{2 \dot{x}}{x}=\frac{\dot{\lambda}}{\lambda+\alpha}+\frac{\dot{\mu}}{\mu+\alpha}+\frac{\dot{v}}{v+\alpha}$, etc.

Therefore we can write

$J=1 / 2 L^{2}-1 / 2(\beta+\gamma) \dot{x}^{2}-1 / 2(\gamma+\alpha) \dot{y}^{2}-1 / 2(\alpha+\beta) \dot{z}^{2}-(\mu+v) \frac{\zeta(\lambda)}{P^{2}}-(v+\lambda) \frac{\eta(\mu)}{Q 2}-(\lambda+\mu) \frac{\theta(v)}{R^{2}}$,

$K=1 / 2 \beta \gamma \dot{x}^{2}+1 / 2 \gamma \alpha \dot{y}^{2}+1 / 2 \alpha \beta \dot{z}^{2}-1 / 2 \alpha L_{x}^{2}-1 / 2 \beta L_{y}^{2}-1 / 2 \gamma L_{z}^{2}-\mu v \frac{\zeta(\lambda)}{P^{2}}-v \lambda \frac{\eta(\mu)}{Q^{2}}-\lambda \mu \frac{\theta(v)}{R^{2}}$,

where $\mathbf{L}=\left(L_{x}, L_{y}, L_{z}\right)=(y \dot{z}-z \dot{y}, z \dot{x}-x \dot{z}, x \dot{y}-y \dot{x})$ is the angular momentum vector, and $L^{2}=L_{x}^{2}+L_{y}^{2}+L_{z}^{2}$. We now write the integrals as

$H=E_{x}+E_{y}+E_{z}$

$J=1 / 2 L^{2}-(\beta+\gamma) E_{x}-(\gamma+\alpha) E_{y}-(\alpha+\beta) E_{z}$,

$K=-1 / 2 \alpha L_{x}^{2}-1 / 2 \beta L_{y}^{2}-1 / 2 \gamma L_{z}^{2}+\beta \gamma E_{x}+\gamma \alpha E_{y}+\alpha \beta E_{z}$,

where

$E_{x}=1 / 2 \dot{x}^{2}+V_{x}$, etc.

and $V_{x}, V_{y}$ and $V_{z}$ must obey

$V_{x}+V_{y}+V_{z}=-\frac{\zeta(\lambda)}{P^{2}}-\frac{\eta(\mu)}{Q^{2}}-\frac{\theta(v)}{R^{2}}=V_{\mathrm{S}}$,

$(\beta+\gamma) V_{x}+(\gamma+\alpha) V_{y}+(\alpha+\beta) V_{z}=(\mu+v) \frac{\zeta(\lambda)}{P^{2}}+(v+\lambda) \frac{\eta(\mu)}{Q^{2}}+(\lambda+\mu) \frac{\theta(v)}{R^{2}}$,

$\beta \gamma V_{x}+\gamma \alpha V_{y}+\alpha \beta V_{z}=-\mu \nu \frac{\zeta(\lambda)}{P^{2}}-v \lambda \frac{\eta(\mu)}{Q^{2}}-\lambda \mu \frac{\theta(v)}{R^{2}}$.

Solving these three equations for $V_{x}$, etc. we find

$V_{x}=-\frac{1}{(\alpha-\gamma)(\alpha-\beta)}\left\{(\mu+\alpha)(v+\alpha) \frac{\zeta(\lambda)}{P^{2}}+(v+\alpha)(\lambda+\alpha) \frac{\eta(\mu)}{Q^{2}}+(\lambda+\alpha)(\mu+\alpha) \frac{\theta(v)}{R^{2}}\right\}$, etc.

$V_{x}, V_{y}$ and $V_{z}$ depend on all three coordinates, but we shall see (Section 3.2) that near the origin $V_{x}$ depends mostly on $x$, etc. This means that $E_{x}, E_{y}$ and $E_{z}$ are the energies in the $x$-, $y$ - and $z$-motion, but only near the origin. 
It is often advantageous to use instead of $J$ and $K$ the integrals $I_{2}$ and $I_{3}$ defined by

$I_{2}=\frac{\alpha^{2} H+\alpha J+K}{\alpha-\gamma}, \quad I_{3}=\frac{\gamma^{2} H+\gamma J+K}{\gamma-\alpha}$.

The following cases are worth spelling out. The integrals on different lines of text are independent.

When $r^{2}=x^{2}+y^{2}+z^{2}$ is much smaller than both $\gamma-\beta$ and $\beta-\alpha$, the ellipsoidal coordinates are nearly Cartesian. In this case $V_{x}$ is a function of $x$ alone, $E_{x}=1 / 2 \dot{x}^{2}+V_{x}(x)$ is the $x$-energy, etc. and $H=E_{x}+E_{y}+E_{z}$,

$J=-(\beta+\gamma) E_{x}-(\gamma+\alpha) E_{y}-(\alpha+\beta) E_{z}, \quad I_{2}=(\alpha-\beta) E_{x}$,

$K=\beta \gamma E_{x}+\gamma \alpha E_{y}+\alpha \beta E_{z}, \quad I_{3}=(\gamma-\beta) E_{z}$.

Clearly, $E_{x}, E_{y}$ and $E_{z}$ are conserved separately. As both $\beta-\alpha \rightarrow \infty$ and $\gamma-\beta \rightarrow \infty$ the coordinates become Cartesian everywhere, and we recover the well-known result that the general potential separable in Cartesian coordinates admits the $x$-, $y$ - and $z$-energies as integrals of motion.

When $\beta=\alpha$ the coordinates become prolate spheroidal coordinates with the $z$-axis as symmetry axis. The general form of $V_{\mathrm{S}}$ in these coordinates is given by Lynden-Bell (1962). For axisymmetric $V_{\mathrm{S}}$ we find

$H=E_{x}+E_{y}+E_{z}$,

$I_{2}=1 / 2 L_{z}^{2}$,

$I_{3}=1 / 2\left(L_{x}^{2}+L_{y}^{2}\right)+(\gamma-\alpha) E_{z}$.

When $\beta=\gamma$ the coordinates are oblate spheroidal coordinates with the $x$-axis as symmetry axis. For axisymmetric $V_{\mathrm{S}}$ we find

$H=E_{x}+E_{y}+E_{z}$,

$I_{2}=1 / 2\left(L_{y}^{2}+L_{z}^{2}\right)+(\alpha-\gamma) E_{x}$,

$I_{3}=1 / 2 L_{x}^{2}$.

Finally, for $\alpha=\beta=\gamma$ the $(\lambda, \mu, v)$-coordinates are spherical coordinates. The general form of $V_{\mathrm{S}}$ is given by Lynden-Bell (1962). For spherical $V_{\mathrm{S}}$ we have

$H=E_{x}+E_{y}+E_{z}$,

$J=1 / 2 L^{2}-2 \alpha H, \quad K=\alpha^{2} H-1 / 2 \alpha L^{2}, \quad I_{2}+I_{3}=1 / 2 L^{2}$.

Clearly, the adelphic integrals $I_{2}$ and $I_{3}$ can be considered as generalizations of the angular momentum integrals that exist in axisymmetric and spherical potentials, but also as generalizations of the energy integrals that exist in potentials separable in Cartesian coordinates.

\subsection{ORBITS}

Every orbit in a Stäckel potential is the sum of three motions, one in each coordinate. As a result, motion is bounded by coordinate surfaces.

In smooth potentials $V_{\mathrm{S}}$ that increase monotonically with increasing radius - which is the case for centrally concentrated mass models - four distinct shapes occur for general orbits. They are called boxes, short axis tubes, inner long axis tubes and outer long axis tubes; they are discussed in detail by de Zeeuw (1985a). The shapes of the volumes allowed to a short axis tube and to an outer long axis tube are illustrated in Fig. 3.

In the present paper we are mainly concerned with finding, for a given non-separable potential $V$, the functions $\zeta(\lambda)$, etc. such that the resulting $V_{\mathrm{S}}$ fits $V$ best. The integrals in $V_{\mathrm{S}}$ then are 


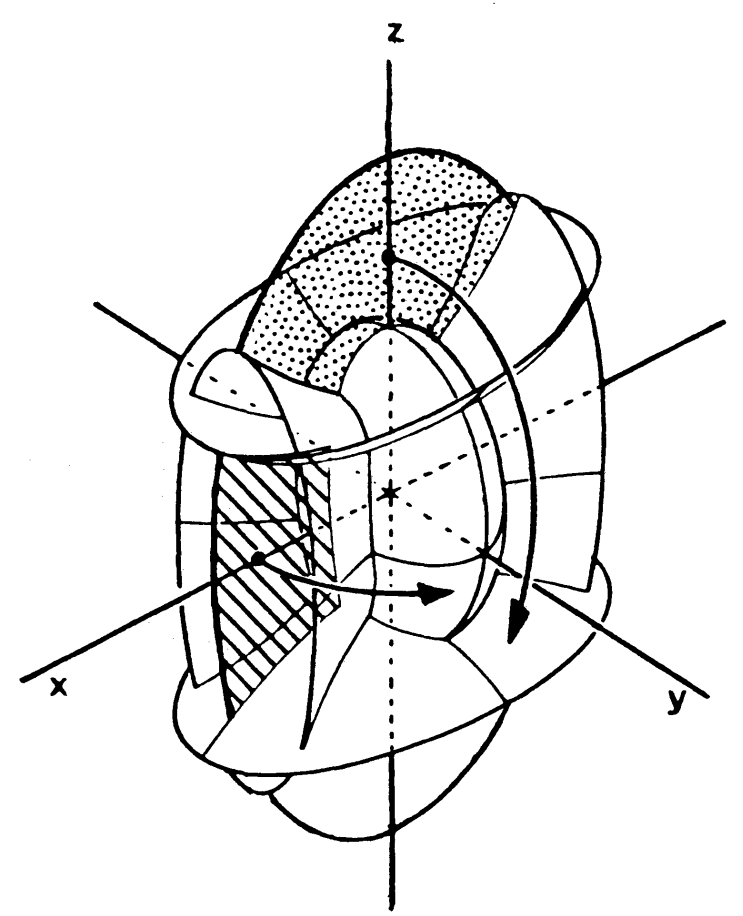

Figure 3. Shapes of two possible three-dimensional orbits in a Stäckel potential. An orbit in the volume bounded by coordinate surfaces that is obtained by taking the dashed area around the $z$-axis, as indicated by the arrow, is a short axis tube orbit. An orbit in the volume that is obtained by taking the dotted area around the $x$-axis in a similar way is an outer long axis tube orbit.

approximations of the integrals in $V$. Also, the orbits in $V$ are approximately those in $V_{\mathrm{S}}$. Since $\zeta(\lambda)$, etc. are determined in the fitting procedure, the orbits in $V_{\mathrm{S}}$ can be found easily by means of the procedure described by de Zeeuw (1985a).

\section{Local fitting}

We expand the potential near an equilibrium point in a stellar system in a power series, and ask to what order this can be fitted term by term with the similar expansion of the general separable potential.

\subsection{EXPANSIONS FOR $V=V\left(x^{2}, y^{2}, z^{2}\right)$}

We expand the potential $V\left(x^{2}, y^{2}, z^{2}\right)$ around the centre as

$V=\sum_{k=0}^{\infty} \sum_{l=0}^{\infty} \sum_{m=0}^{\infty} V_{k l m} x^{k} y^{l} z^{m}$

where only even values of $k, l$ and $m$ occur. We compare this with the similar expansion of a separable potential of the form (2). The origin is at $(\lambda, \mu, v)=(-\alpha,-\beta,-\gamma)$, and we expand $\zeta(\lambda)$, $\eta(\mu)$ and $\theta(v)$ as

$\zeta(\lambda)=\sum_{n=0}^{\infty} \zeta_{n}(\lambda+\alpha)^{n}, \eta(\mu)=\sum_{n=0}^{\infty} \eta_{n}(\mu+\beta)^{n}, \theta(v)=\sum_{n=0}^{\infty} \theta_{n}(v+\gamma)^{n}$.

For $V_{\mathrm{S}}$ to be regular at the origin, $\zeta^{*}(\lambda), \eta^{*}(\mu)$ and $\theta^{*}(v)$ must be. Hence, $\zeta(\lambda)$ may start its expansion with a term $\zeta_{-1} /(\lambda+\alpha)$; similarly, the expansion of $\eta(\mu)$ may start with $\eta_{-1} /(\mu+\beta)$ and that of $\theta(v)$ with $\theta_{-1} /(v+\gamma)$. However, we may add $\left(c_{1}+c_{2} \lambda\right)(\lambda+\alpha)(\lambda+\beta)(\lambda+\gamma)$ to $\zeta(\lambda)$, and similar terms to $\eta(\mu)$ and $\theta(v)$, where $c_{1}$ and $c_{2}$ are arbitrary constants, without changing $V_{\mathbf{S}}$. Thus, we may choose $c_{1}$ and $c_{2}$ such that $\zeta_{-1}=\eta_{-1}=\theta_{-1}$. If we then also choose $V$ (and $V_{\mathrm{S}}$ ) to be 
zero at the origin we may take, without loss of generality, $\zeta_{-1}=\eta_{-1}=\theta_{-1}=0$. Then we find

$$
\begin{aligned}
\frac{\zeta(\lambda)}{P^{2}}= & 4 x^{2}\left\{\zeta_{0}+\zeta_{1} x^{2}+\frac{2 \zeta_{0}}{(\beta-\alpha)} y^{2}+\frac{2 \zeta_{0}}{(\gamma-\alpha)} z^{2}+\zeta_{2} x^{4}+\frac{3 \zeta_{1}(\beta-\alpha)-3 \zeta_{0}}{(\beta-\alpha)^{2}} x^{2} y^{2}\right. \\
& \left.+\frac{3 \zeta_{1}(\gamma-\alpha)-3 \zeta_{0}}{(\gamma-\alpha)^{2}} y^{2} z^{2}+\frac{3 \zeta_{0}}{(\beta-\alpha)^{2}} y^{4}+\frac{6 \zeta_{0}}{(\beta-\alpha)(\gamma-\alpha)} y^{2} z^{2}+\frac{3 \zeta_{0}}{(\gamma-\alpha)^{2}} z^{4}+\ldots\right\}, \text { etc. }
\end{aligned}
$$

where we have used

$$
\begin{aligned}
& \lambda+\alpha=x^{2}\left\{1+\frac{y^{2}}{\beta-\alpha}+\frac{z^{2}}{\gamma-\alpha}-\frac{y^{2}\left(x^{2}-y^{2}\right)}{(\beta-\alpha)^{2}}+\frac{2 y^{2} z^{2}}{(\beta-\alpha)(\gamma-\alpha)}-\frac{z^{2}\left(x^{2}-z^{2}\right)}{(\gamma-\alpha)^{2}}+\ldots\right\}, \text { etc. } \\
& \frac{1}{P^{2}}=4 x^{2}\left\{1+\frac{2 y^{2}}{\beta-\alpha}+\frac{2 z^{2}}{\gamma-\alpha}-\frac{3 y^{2}\left(x^{2}-y^{2}\right)}{(\beta-\alpha)^{2}}+\frac{6 y^{2} z^{2}}{(\beta-\alpha)(\gamma-\alpha)}-\frac{3 z^{2}\left(x^{2}-z^{2}\right)}{(\gamma-\alpha)^{2}}+\ldots\right\}, \text { etc. }
\end{aligned}
$$

For the sum $P^{-2} \zeta(\lambda)+Q^{-2} \eta(\mu)+R^{-2} \theta(v)$ we then obtain an expansion of the form (19) with

$$
\begin{array}{ll}
V_{000}=0, & V_{200}=-4 \zeta_{0}, \\
V_{400}=-4 \zeta_{1}, & V_{220}=-\frac{8}{(\beta-\alpha)}\left(\zeta_{0}-\eta_{0}\right), \\
V_{600}=-4 \zeta_{2}, & V_{420}=-\frac{12}{(\beta-\alpha)^{2}}\left[\zeta_{1}(\beta-\alpha)-\zeta_{0}+\eta_{0}\right], \\
V_{222}=\frac{24\left[\zeta_{0}(\gamma-\beta)+\eta_{0}(\alpha-\gamma)+\theta_{0}(\beta-\alpha)\right]}{(\gamma-\beta)(\beta-\alpha)(\alpha-\gamma)},
\end{array}
$$

where the other coefficients up to sextic order are given by symmetry.

Equations (23) may be used to determine $\zeta_{n}, \eta_{n}$ and $\theta_{n}$ as well as $\beta-\alpha$ and $\gamma-\beta$ for given expansion coefficients $V_{k l m}$. This yields the separable potential $V_{\mathrm{S}}$ that fits the given potential $V$ term by term.

To order $2 n$ in the displacements $x, y$ and $z$ there are $1 / 6(n+1)(n+2)(n+3)-1$ coefficients in the expansion (19) (taking $V_{000}=0$ ). In the separable potential there are $3 n+2$ parameters: $\zeta_{0}$, $\ldots, \zeta_{n-1}$ give $n$ as do $\eta$ and $\theta$, and to these we must add $(\beta-\alpha)$ and $(\gamma-\beta)$ which define the ellipsoidal coordinates. Thus, at order $2 n$ we expect $(n+1)[1 / 6(n+2)(n+3)-3]$ relations between the coefficients of the separable potential (provided that number is positive). These relations are constraints in the fitting. At quartic order $(n=2)$ this gives one constraint which is easily seen to be

$\frac{V_{200}-V_{020}}{V_{220}}+\frac{V_{020}-V_{002}}{V_{022}}+\frac{V_{002}-V_{200}}{V_{202}}=0$.

Thus, in three dimensions the general quartic with three reflection symmetries cannot be fitted with a separable potential.

We show in Section 4 that the constraint (24) is automatically satisfied by the quartic terms of the potentials of similar stratified ellipsoids, and also by potentials that are constant on similar ellipsoids, among many others.

\subsection{THE ADELPHIC INTEGRALS}

Substitution of the expansions (21) and (22) in the expressions for $V, V_{y}$ and $V_{z}$ given in (12) 
produces

$V_{x}=\sum_{k=0}^{\infty} \sum_{l=0}^{\infty} \sum_{m=0}^{\infty} \frac{k}{k+l+m} V_{k l m} x^{k} y^{l} z^{m}$

$V_{y}=\sum_{k=0}^{\infty} \sum_{l=0}^{\infty} \sum_{m=0}^{\infty} \frac{l}{k+l+m} V_{k l m} x^{k} y^{l} z^{m}$

$V_{z}=V-V_{x}-V_{y}$,

where only even values of $k, l$ and $m$ occur. Substitution in (10) gives expansions for the integrals of motion directly in terms of the expansion coefficients $V_{k l m}$ of the potential $V$. Thus, one may write down approximate expressions for the integrals of motion without actually calculating the separable expansion that fits $V$ best.

With $1 /(\beta-\alpha), 1 /(\gamma-\alpha)$ and $1 /(\gamma-\beta)$ occurring in many places, it is evident that the expansions in Section 3.1 have radii of convergence of at most order $\min (\gamma-\beta, \beta-\alpha)$ by assumption $(\gamma-\alpha) \geq(\gamma-\beta)-$ see Section 2.1 $)$. The expansion for $V_{x}$ may have a different radius of convergence, equal to that of the expansion (19) for $V$. At large distances from the origin the expansions will not be valid, but this does not necessarily mean that $V$ cannot be approximated there by a Stäckel potential $V_{\mathrm{S}}$. We return to this point in Sections 4 and 5 .

We emphasize that we have only found an approximate expansion for the integrals of motion in $V$. Our results are formal: the accuracy of the approximate integrals cannot be established from the fitting alone. This problem is addressed in a companion paper (de Zeeuw 1985b), where we delineate the precise circumstances under which local fitting of $V\left(x^{2}, y^{2}, z^{2}\right)$ produces rigorous results.

We shall see in Section 4 that local fitting can in some cases lead to the discovery of interesting potentials that are exactly, i.e. globally, separable.

It is worth mentioning a few special cases. When $V_{220}=V_{202}=V_{022}=0$, the foci of the ellipsoidal coordinates are at infinity, so that the coordinates are Cartesian. In this case $V_{\mathrm{S}}$ is of the form $-\left\{\zeta\left(x^{2}\right)+\eta\left(y^{2}\right)+\theta\left(z^{2}\right)\right\}$, and we can fit the quartic terms. The integrals are the energies $E_{x}, E_{y}$ and $E_{z}(c f$. Section 2.2).

When $V_{220}=V_{202}=V_{022}$, and the coefficients of the mixed quartic terms are non-zero, the condition (24) is satisfied. However, now $\beta-\alpha=\gamma-\alpha=\gamma-\beta=0$. This means that the ellipsoidal coordinates are spherical coordinates. The general potential separable in spherical coordinates is regular at the origin only if it is of the form $V_{\mathrm{S}}=V_{\mathrm{S}}\left(x^{2}+y^{2}+z^{2}\right)$. As a result, local fitting is to be done with a spherical potential, and there is one adjustable parameter at each order. Thus, we can in general fit only one of the quartic terms. The integrals of motion in $V_{\mathrm{S}}$ are $E$ and $\mathbf{L}=\left(L_{x}, L_{y}\right.$, $\left.L_{z}\right)$.

When $V_{200}=V_{020}$ and $V_{220} \neq 0$, then $\beta=\alpha$ and the coordinates are prolate spheroidal coordinates. $V_{\mathrm{S}}$ is regular at the origin if it is axisymmetric, i.e. $V_{\mathrm{S}}=V_{\mathrm{S}}\left(x^{2}+y^{2}, z^{2}\right)$. It then follows that we can fit the quadratic terms and three of the quartic terms of a general potential $V\left(x^{2}, y^{2}\right.$, $\left.z^{2}\right)$. In this case the integrals of motion are $E, 1 / 2 L_{z}^{2}$ and $1 / 2\left(L_{x}^{2}+L_{y}^{2}\right)+(\gamma-\alpha) E_{z}$.

\subsection{POTENTIALS WITH FEWER SYMMETRIES}

When the potential has only two planes of symmetry, i.e. $V=V\left(x^{2}, y^{2}, z\right)$, then we may expand it about an equilibrium point (which we take as the origin) in the form

$$
\begin{aligned}
V= & V_{200} x^{2}+V_{020} y^{2}+V_{002} z^{2}+V_{201} x^{2} z+V_{021} y^{2} z+V_{003} z^{3} \\
& +V_{400} x^{4}+V_{220} x^{2} y^{2}+V_{202} x^{2} z^{2}+V_{040} y^{4}+V_{022} y^{2} z^{2}+V_{004} z^{4}+\ldots
\end{aligned}
$$


We compare this expansion with that of a separable potential $V_{\mathrm{S}}$ around some point $\left(0,0, z_{0}\right)$ where we make $V_{\mathrm{S}}$ to have an equilibrium point. We shall only count the number of parameters that may be fitted and determine the number of constraints on the higher orders.

First consider $V$. At order $2 n$ and at order $2 n+1$ there are the same number of terms: $1 / 2(n+1)(n+2)$. Therefore the total number of terms up to order $2 n$ is $1 / 6(n+1)(n+2)(2 n+3)-1$ and up to order $2 n+1$ is $1 / 3(n+1)(n+2)(n+3)-2$.

For the expansion of $V_{\mathrm{S}}$ we take $\zeta(\lambda), \eta(\mu)$ and $\theta(v)$ in the form (20) but with $\left(v+\gamma-z_{0}^{2}\right)$ instead of $(v+\gamma)$. With $V_{000}=0$, we can take $\zeta_{-1}=\eta_{-1}=\theta_{-1}=0$ without loss of generality, as in Section 3.1. We then have $2 n$ parameters $\zeta_{i}, \eta_{i}(i=0, \ldots, n-1)$ and $2 n$ parameters $\theta_{i}(i=0, \ldots, 2 n-1)$ to which we should add another two for the interfocal distances. There is one arbitrary choice in $z_{0}$ but one extra constraint that $\partial V_{\mathrm{S}} / \partial z$ be zero there. Thus, at order $2 n$ there are $4 n+2$ adjustable parameters. At order $2 n+1$ there is just one more, $\theta_{2 n}$. This means that at order 3 we have 6 terms in the general expansion while there are 7 free coefficients, so the potential can be fitted to cubic order. However, at order 4 there will be two constraints.

The similar enumerations for potentials with only one plane of symmetry or with no plane of symmetry are given in Table 1 . For each case it gives the number $C$ of coefficients in $V$, the number $P$ of adjustable parameters in $V_{\mathrm{S}}$ and the number $C-P$ of constraints, all as a function of the order of the terms. $V_{n}$ indicates the terms of order $n$. In the counting we have assumed that the mixed quadratic terms $\left[y z\right.$ for $V\left(x^{2}, y, z\right)$ and $x y, x z$ and $y z$ for $\left.V(x, y, z)\right]$ are all zero. This can always be achieved by a rotation of the coordinate system. We conclude that for potentials with no symmetry it is generally not even possible to fit the cubic terms with a separable potential.

The results given in Table 1 are valid for a general expansion of a three-dimensional potential $V$. In special cases the equations that relate the coefficients $V_{k l m}$ to the adjustable parameters may not be all independent. Such cases have to be treated separately. Furthermore, the results are formal; the accuracy of the approximations for the integrals of motion is not established.

\section{Two applications}

We apply the results obtained in Section 3 to expansions of potentials of ellipsoidally stratified density distributions and to ellipsoidal potentials. We show that the constraint (24) is satisfied for

Table 1. Number of adjustable parameters when fitting a three-dimensional potential with a Stäckel potential.

\begin{tabular}{|c|c|c|c|c|}
\hline CASE & ORDER & C & $\mathbf{p}$ & C-P \\
\hline \multirow[t]{2}{*}{$\mathrm{V}\left(\mathrm{x}^{2}, \mathrm{y}^{2}, \mathrm{z}^{2}\right)$} & $\mathrm{v}_{2}$ & 3 & 5 & - \\
\hline & $v_{4}$ & 9 & 8 & $1^{*}$ \\
\hline \multirow[t]{3}{*}{$\mathrm{V}\left(\mathrm{x}^{2}, \mathrm{y}^{2}, \mathrm{z}\right)$} & $\mathrm{v}_{2}$ & 3 & 6 & - \\
\hline & $v_{3}$ & 6 & 7 & - \\
\hline & $\mathrm{v}_{4}$ & 12 & 10 & 2 \\
\hline \multirow[t]{3}{*}{$\mathrm{V}\left(\mathrm{x}^{2}, \mathrm{y}, \mathrm{z}\right)$} & $\mathrm{v}_{2}$ & 3 & 7 & - \\
\hline & $v_{3}$ & 9 & 9 & 0 \\
\hline & $\mathrm{v}_{4}$ & 18 & 12 & 6 \\
\hline \multirow[t]{2}{*}{$V(x, y, z)$} & $\mathrm{v}_{2}$ & 3 & 8 & - \\
\hline & $v_{3}$ & 13 & 11 & 2 \\
\hline
\end{tabular}


all these potentials and consider the question of agreement with a Stäckel potential to higher order.

\subsection{THE GRAVITATIONAL POTENTIAL OF ELLIPSOIDAL DENSITY DISTRIBUTIONS}

Consider a triaxial density distribution stratified on similar, concentric ellipsoids. The density can be written as $\varrho=\varrho\left(\mathrm{m}^{2}\right)$ where

$m^{2}=\frac{x_{1}^{2}}{a_{1}^{2}}+\frac{x_{2}^{2}}{a_{2}^{2}}+\frac{x_{3}^{2}}{a_{3}^{2}}, \quad a_{1} \geq a_{2} \geq a_{3} \geq 0$.

Here we have written $x=x_{1}, y=x_{2}$ and $z=x_{3}$, and we have assumed that the long axis of the density distribution is in the $x$-direction, and the short axis in the $z$-direction. The gravitational potential $V$ of such an ellipsoidally stratified mass distribution can be found by classical theory (e.g. Chandrasekhar 1969, hereafter EFE).

We are interested in cases in which $V$ is analytic near the centre. This means that near the centre the density $\varrho\left(\mathrm{m}^{2}\right)$ has the Taylor expansion

$\varrho\left(m^{2}\right)=\varrho_{0}\left(1+\alpha_{1} m^{2}+\alpha_{2} m^{4}+\ldots\right)=\varrho_{0} \sum_{n=0}^{\infty} \alpha_{n} m^{2 n}$.

With the help of Theorem 12 from Chapter 3 of EFE we find for the potential $V$ of the density distribution (28)

$V=-\pi G \varrho_{0} \sum_{n=0}^{\infty} \frac{\alpha_{n}}{n+1}\left\{I-\sum_{i_{p}=1}^{3} A_{i_{1} i_{2} \ldots i_{n+1}} x_{i_{1}}^{2} x_{i_{2}}^{2} \ldots x_{i_{n+1}}^{2}\right\}$

where the $i_{p}$ sum is over all $i_{p}$ from $p=1$ to $n+1$. The constant $I$, and the index symbols $A_{i_{1} i_{2} \ldots i_{n}}$ are defined in EFE (p. 54). It is convenient to express the index symbols in terms of the lower order symbols. If not all indices are identical, one may use the relation (EFE)

$A_{i j k l \ldots}=-\frac{\left(A_{i k l \ldots}-A_{j k l \ldots}\right)}{\left(a_{i}^{2}-a_{j}^{2}\right)}, \quad i \neq j$.

The remaining symbols with all indices identical can then be found from

$(2 n-1) A_{i i i \ldots i i}+A_{i i i \ldots i j}+A_{i i i \ldots i k}=\frac{2}{a_{i}^{2 n-2}}$,

where $i, j$ and $k$ are all different and the index symbols occurring in this equation are of order $n$. In EFE the index symbols of order one are given explicitly in terms of incomplete elliptic integrals of the first and second kinds.

We now investigate how well the potential (29) can be fitted by a separable one of the form discussed in Section 3. The condition (24) for separability up to and including quartic terms, when applied to the expansion (29) reads

$\frac{1}{\alpha_{1}}\left\{\frac{A_{1}-A_{2}}{A_{12}}+\frac{A_{2}-A_{3}}{A_{23}}+\frac{A_{3}-A_{1}}{A_{31}}\right\}=0$.

With the aid of (30) the left-hand side of this equation becomes

$\left(a_{1}^{2}-a_{2}^{2}+a_{2}^{2}-a_{3}^{2}+a_{3}^{2}-a_{1}^{2}\right) / \alpha_{1}$,

which is certainly zero. So the potentials of stratified ellipsoids satisfy the constraint (24) and agree 
with separable potentials $V_{S}$ up to and including quartic order. This is true for arbitrary values of $\alpha_{1}$. The Stäckel potential $V_{\mathrm{S}}$ that fits (29) to this order is defined in terms of its expansion coefficients $V_{k l m}$ given in (23). We choose $\zeta_{0}=-\pi G \varrho_{0} A_{1} / 4, \zeta_{1}=-\pi G \varrho_{0} \alpha_{1} A_{11} / 8$, etc. The ellipsoidal coordinate system is now also defined. We require

$\beta-\alpha=-\frac{2}{\alpha_{1}}\left(a_{1}^{2}-a_{2}^{2}\right), \quad \gamma-\alpha=-\frac{2}{\alpha_{1}}\left(a_{1}^{2}-a_{3}^{2}\right), \quad \gamma-\beta=-\frac{2}{\alpha_{1}}\left(a_{2}^{2}-a_{3}^{2}\right)$,

so that we may take

$\alpha=2 a_{1}^{2} / \alpha_{1}, \quad \beta=2 a_{2}^{2} / \alpha_{1}, \quad \gamma=2 a_{3}^{2} / \alpha_{1}$.

The above result raises the question of agreement to higher order. First consider the sextic terms of $V$. We can fit the coefficients of $x_{1}^{6}, x_{2}^{6}$ and $x_{3}^{6}$ by taking $\zeta_{2}=-\pi G \varrho_{0} \alpha_{2} A_{111} / 12$ and similar expressions for $\eta_{2}$ and $\theta_{2}$. The remaining mixed sextic terms in the separable expansion (23) are now fixed, and can be rewritten, with the help of (30), as follows

$3 / 4 \alpha_{1}^{2} \pi G \varrho_{0}\left(A_{112} x_{1}^{4} x_{2}^{2}+A_{113} x_{1}^{4} x_{3}^{2}+A_{122} x_{1}^{2} x_{2}^{4}+2 A_{123} x_{1}^{2} x_{2}^{2} x_{3}^{2}+A_{133} x_{1}^{2} x_{3}^{4}+A_{223} x_{2}^{4} x_{3}^{2}+A_{233} x_{2}^{2} x_{3}^{4}\right)$.

A comparison with (29) shows that the ratios of the mixed sextic terms of $V_{\mathrm{S}}$ are identical to those of $V$. In general, however, they will differ by a constant factor. Only for the case where $\alpha_{2}=3 / 4 \alpha_{1}^{2}$ does $V_{\mathrm{S}}$ fit $V$ up to and including sextic terms.

A similar result holds for all higher orders. One can always fit the coefficients $[1 /(n+1)] \alpha_{n} A_{i \ldots i}$ of $x_{i}^{2 n+2}$ by the three adjustable parameters that now occur in $V_{\mathrm{S}}$. The mixed terms of this order are now all determined, but only their ratios are the same as in $V$. Assuming one has fitted all terms to order $2 n$ in $x_{1}, x_{2}$ and $x_{3}$, then all terms of order $2 n+2$ can be fitted only if

$\alpha_{n}=\frac{n+1}{2 n} \alpha_{n-1} \alpha_{1}$.

The curious conclusion must be drawn that one form for $\varrho\left(\mathrm{m}^{2}\right)$ exists for which $V_{S}$ fits its potential $V$ to all orders. From equations (28) and (34) we find for this density

$\varrho\left(m^{2}\right)=\varrho_{\mathrm{sep}}\left(m^{2}\right)=\varrho_{0} \sum_{n=0}^{\infty} \frac{n+1}{2^{n}} \alpha_{1}^{n} m^{2 n}$

This is the series expansion for small $\mathrm{m}^{2}$ of the following density profile

$\varrho_{\text {sep }}\left(m^{2}\right)=\frac{\varrho_{0}}{\left(1-1 / 2 \alpha_{1} m^{2}\right)^{2}}$.

For densities that are centrally concentrated $\alpha_{1}<0$. By a simple rescaling we can make $\alpha_{1}=-2$. The normalized density thus becomes

$\varrho_{\text {sep }}\left(m^{2}\right)=\frac{\varrho_{0}}{\left(1+m^{2}\right)^{2}}$

We conclude that with this unique density profile, a stratified ellipsoid with any axial ratios has a potential of Stäckel form, so that the equations of motion are separable in ellipsoidal coordinates $(\lambda, \mu, v)$ defined by $\alpha=-a_{1}^{2}, \beta=-a_{2}^{2}$ and $\gamma=-a_{3}^{2}$ \{see equation (33) $\}$. Note that, although the series expansion (28) is valid only for $0 \leq m^{2} \leq 1$, the density (36) is defined for all $m^{2} \geq 0$. The potential is of Stäckel form at all radii, and the integrals are exact everywhere. We shall refer to the mass model (36) as the perfect ellipsoid.

Motion in the perfect ellipsoid is tractable by analytic means; it is explored in detail in a paper 
entitled 'Elliptical Galaxies with Separable Potentials' (de Zeeuw 1985a). The axially symmetric case $a_{1}=a_{2}$ was discovered by Kuzmin (1956) in his classic study of separable mass models for the Galaxy.

\subsection{ELLIPSOIDAL POTENTIALS}

The wide class of symmetric potentials that satisfy the constraint (24) for separability up to and including quartic order not only contains the potentials of densities $\varrho=\varrho\left(\mathrm{m}^{2}\right)$, but also contains all potentials that are analytic in the centre whose equipotential surfaces are similar concentric ellipsoids, i.e. $V=V\left(\mathrm{~m}^{2}\right)$ with $\mathrm{m}^{2}$ given in (27). Among these there is one that is separable to all orders:

$$
\begin{aligned}
V=\frac{V_{0}}{1+m^{2}} & =\frac{A V_{0}}{(\lambda+\delta)(\mu+\delta)(v+\delta)} \\
& =A V_{0}\left\{\frac{1 / \lambda+\delta}{(\lambda-\mu)(\lambda-v)}+\frac{1 / \mu+\delta}{(\mu-v)(\mu-\lambda)}+\frac{1 / v+\delta}{(v-\lambda)(v-\mu)}\right\},
\end{aligned}
$$

where $A=(\delta-\alpha)(\delta-\beta)(\delta-\gamma), V_{0}$ is a constant and the connection between the $\alpha, \beta$ and $\gamma$ of the ellipsoidal coordinates and the $a_{1}^{2}, a_{2}^{2}$ and $a_{3}^{2}$ of $m^{2}$ is given by

$a_{1}^{2}=\delta-\alpha, \quad a_{2}^{2}=\delta-\beta, \quad a_{3}^{2}=\delta-\gamma$.

Notice that the value of $\delta$ is arbitrary. Without loss of generality $\delta$ may be taken equal to zero.

The potential (37) evidently has a simple form in Cartesian coordinates. This is also true for the adelphic integrals $J$ and $K$. They are given by equations (10) and

$V_{x}=V_{0} \frac{\left\{\left(a_{2}^{2}+a_{3}^{2}-a_{1}^{2}\right) x_{1}^{2}+a_{3}^{2} x_{2}^{2}+a_{2}^{2} x_{3}^{2}+a_{2}^{2} a_{3}^{2}\right\}}{\left(a_{1}^{2}-a_{2}^{2}\right)\left(a_{1}^{2}-a_{3}^{2}\right)\left\{1+x_{1}^{2} / a_{1}^{2}+x_{2}^{2} / a_{2}^{2}+x_{3}^{2} / a_{3}^{2}\right\}}$, etc.

Although the motion in this potential is obviously best described in the coordinates $(\lambda, \mu, v)$ in which the equations of motion separate, the relative simple form of the integrals in Cartesian coordinates allows one to infer various properties of the orbits without using the ellipsoidal coordinates. Unfortunately, the potential (37) dies away too fast at infinity to correspond to a sensible mass model. It may however be used as a simple example of an exactly separable potential.

\section{Global fitting}

The example of the perfect ellipsoid described in Section 4.1 shows that potentials relevant for elliptical galaxies can be well approximated by the Stäckel form not only locally, but also globally. We describe a method for finding a global separable fit to a given potential $V\left(x^{2}, y^{2}, z^{2}\right)$.

5.1 CALCulation of $\zeta^{*}(\lambda), \eta^{*}(\mu)$ and $\theta^{*}(v)$

Consider a potential $V\left(x^{2}, y^{2}, z^{2}\right)$. We wish to approximate $V$ by a Stäckel potential $V_{\mathrm{S}}$. Our task is to determine an ellipsoidal coordinate system, and the functions $\zeta^{*}(\lambda), \eta^{*}(\mu)$ and $\theta^{*}(v)$, in such a way that (2) fits $V$ globally as closely as possible. It is clear that a procedure for doing this should involve some sort of averaging, and should give the correct answer if $V$ happens to be of exactly Stäckel form. The following method has both these properties.

First pick an ellipsoidal coordinate system $(\lambda, \mu, v)$ and define the following averages for any 
function $Q(\lambda, \mu, v)$

$$
\begin{aligned}
& \langle Q, \lambda\rangle=\frac{1}{M N} \int_{-\beta}^{-\alpha} \int_{-\gamma}^{-\beta} Q(\lambda, \mu, v) M(\mu) N(v) d \mu d v \\
& \langle Q, \mu\rangle=\frac{1}{\Lambda N} \int_{-\alpha}^{-\infty} \int_{-\gamma}^{-\beta} Q(\lambda, \mu, v) \Lambda(\lambda) N(v) d \lambda d v \\
& \langle Q, v\rangle=\frac{1}{\Lambda M} \int_{-\alpha}^{-\infty} \int_{-\beta}^{-\alpha} Q(\lambda, \mu, v) \Lambda(\lambda) M(\mu) d \lambda d \mu \\
& \bar{Q}=\frac{1}{\Lambda M N} \int_{-\alpha}^{-\infty} \int_{-\beta}^{-\alpha} \int_{-\gamma}^{-\beta} Q(\lambda, \mu, v) \Lambda(\lambda) M(\mu) N(v) d \lambda d \mu d v
\end{aligned}
$$

Here $\Lambda(\lambda), M(\mu)$ and $N(v)$ are weighting functions chosen subject to the constraints that the integrals

$$
\Lambda=\int_{-\alpha}^{\infty} \Lambda(\lambda) d \lambda, \quad M=\int_{-\beta}^{-\alpha} M(\mu) d \mu, \quad N=\int_{-\gamma}^{-\beta} N(v) d v
$$

are finite. The constants $\alpha, \beta$ and $\gamma$ define the ellipsoidal coordinates.

Transform the given potential $V\left(x^{2}, y^{2}, z^{2}\right)$ to $V(\lambda, \mu, v)$ by means of equation (3), and calculate the auxiliary function $\chi$ defined by

$$
\chi(\lambda, \mu, v)=-(\lambda-\mu)(\mu-v)(v-\lambda) V(\lambda, \mu, v) .
$$

Then calculate $\zeta^{*}(\lambda), \eta^{*}(\mu)$ and $\theta^{*}(v)$ from

$$
\begin{aligned}
& \zeta^{*}(\lambda)=\frac{\langle\chi-2 / 3 \bar{\chi}, \lambda\rangle}{(\bar{v}-\bar{\mu})}+\frac{\bar{\chi}(\bar{\lambda}-\lambda)(2 \bar{\lambda}-\bar{\mu}-\bar{v})}{3 L}, \\
& \eta^{*}(\mu)=\frac{\langle\chi-2 / 3 \bar{\chi}, \mu\rangle}{(\bar{\lambda}-\bar{v})}+\frac{\bar{\chi}(\bar{\mu}-\mu)(2 \bar{\mu}-\bar{v}-\bar{\lambda})}{3 L}, \\
& \theta^{*}(v)=\frac{\langle\chi-2 / 3 \bar{\chi}, v\rangle}{(\bar{\mu}-\bar{\lambda})}+\frac{\bar{\chi} \bar{\nu} \mid \bar{v}-v)(2 \bar{v}-\bar{\lambda}-\bar{\mu})}{3 L},
\end{aligned}
$$

where

$L=(\bar{\lambda}-\bar{\mu})(\bar{\mu}-\bar{v})(\bar{v}-\bar{\lambda})$.

This is the desired result. Equation (43) gives the functions $\xi^{*}(\lambda), \eta^{*}(\mu)$ and $\theta^{*}(v)$ that define the Stäckel potential $V_{\mathrm{S}}$ that fits the given $V$ in an average sense in the chosen ellipsoidal coordinates. In order to optimize the fit, one may repeat the process for different choices of the $(\lambda, \mu, v)$-coordinates. We consider the choice of the foci below.

If $V$ happens to be of Stäckel form in the chosen ellipsoidal coordinates, (43) gives the correct result. If $V$ equals $V_{\mathrm{S}}$ in some other $(\lambda, \mu, v)$-coordinate system, then, by repetition of the procedure, one will eventually find a coordinate system in which the fit is exact.

So far, we have not specified the weighting functions $\Lambda(\lambda), M(\mu)$ and $N(v)$. Obviously, if $V=V_{\mathrm{S}}$ then their choice is unimportant. For the general case where $V \neq V_{\mathrm{S}}$ the weighting functions may be chosen to put the emphasis on the fitting where it is most important, e.g. by making the potential fit best where most of the density is. 


\subsection{DETERMINATION OF THE ELLIPSOIDAL COORDINATES}

The ellipsoidal coordinates reduce to elliptic coordinates in each of the principal planes $z=0$, $y=0$ and $x=0$. Thus, there are three pairs of foci: one pair on the $y$-axis, at $y= \pm \sqrt{\beta-\alpha}$, and two pairs on the $z$-axis, at $z= \pm \sqrt{\gamma-\beta}$ and at $z= \pm \sqrt{\gamma-\alpha}$. The ellipsoidal coordinate system is determined by specification of two of these positions, e.g. by giving $\beta-\alpha$ and $\gamma-\beta$. The third position then follows from $\gamma-\alpha=(\gamma-\beta)+(\beta-\alpha)$. We mention three methods for finding approximate positions of the foci. They can be useful for obtaining good initial guesses for these positions in the fitting procedure.

(i) Local fitting. If one knows an expansion of the given potential $V$ around the centre, one may determine $\beta-\alpha$ and $\gamma-\beta$ from $\beta-\alpha=2\left(V_{200}-V_{020}\right) / V_{220}$ and $\gamma-\beta=2\left(V_{020}-V_{002}\right) / V_{022}[c f$. (23)]. The value of $\gamma-\alpha$ is now fixed, but is not necessarily equal to $2\left(V_{002}-V_{200}\right) / V_{202}$. This is only the case if the condition (24) for separability up to quartic order is met. Nevertheless, the values for any two of the interfocal distances should give a good initial guess for the coordinate system to be used in the global fitting.

(ii) Graphical method. If we differentiate $(\lambda-\mu)(\lambda-v) V_{\mathrm{S}}$ with respect to $\lambda$, etc. and then compare the values of the resulting three functions at the origin and at the three pairs of loci we find

$$
\begin{aligned}
& \left.\frac{\partial V_{\mathrm{S}}}{\partial x^{2}}\right|_{(0,0,0)}=\frac{V_{\mathrm{S}}(0, \beta-\alpha, 0)-V_{\mathrm{S}}(0,0,0)}{\beta-\alpha}, \\
& \left.\frac{\partial V_{\mathrm{S}}}{\partial y^{2}}\right|_{(0,0,0)}=\frac{V_{\mathrm{S}}(0,0, \gamma-\beta)-V_{\mathrm{S}}(0,0,0)}{\gamma-\beta}, \\
& \left.\frac{\partial V_{\mathrm{S}}}{\partial z^{2}}\right|_{(0,0,0)}=\frac{V_{\mathrm{S}}(0,0, \gamma-\alpha)-V_{\mathrm{S}}(0,0,0)}{\gamma-\alpha} .
\end{aligned}
$$

Now determine the value $g$, say, of $\partial V_{\mathrm{S}} / \partial x^{2}$ at $(0,0,0)$, and then plot a graph of $\Psi=V_{\mathrm{S}}\left(0, y^{2}\right.$, $0)-V_{\mathrm{S}}(0,0,0)$ versus $y^{2}$. Where it crosses $\Psi=g y^{2}$ we have $y^{2}=\beta-\alpha$. This same construction can, of course, be used in a non-separable potential $V$ so that one can easily find an approximate value for $\beta-\alpha$. The value of $\gamma-\beta$ can be found in a similar manner. Again, the third interfocal distance is now fixed, and is generally not equal to what the third equation in (45) would give.

(iii) Stability analysis of axial orbits. In a potential $V\left(x^{2}, y^{2}, z^{2}\right)$ the oscillations along the principal axes are solutions of the equations of motion. This is true a forteriori for Stäckel potentials $V_{\mathrm{S}}(\lambda, \mu, v)$. For these potentials the foci of the ellipsoidal coordinates coincide with the bifurcation points of the $y$ - and $z$-axis oscillations (de Zeeuw 1985a). At these points the axial oscillations become unstable to perpendicular perturbations, and a family of elliptic closed orbits branches off (Fig. 4).

A non-separable potential $V\left(x^{2}, y^{2}, z^{2}\right)$ does not have foci. However, the periodic orbits that exist in $V_{\mathrm{S}}$ are also found in Schwarzschild's non-separable potential (Schwarzschild 1979, 1981; Heiligman \& Schwarzschild 1979; de Zeeuw \& Merritt 1983). The basic periodic orbits in Schwarzschild's potential are exactly the six that occur in $V_{\mathrm{S}}$ (three axial oscillations and three elliptic closed orbits, one in each principal plane), and with the same stability properties. In particular, there is one pair of bifurcation points on the intermediate $(y)$ axis, and there are two such pairs on the short $(z)$ axis. Thus, we can use the positions of the bifurcation points in the potential $V$ as approximate positions of the foci.

In general the three pairs of bifurcation points may not satisfy the conditions on the positions of the foci mentioned earlier. A good choice is probably to derive $\beta-\alpha$ from the distance to the 


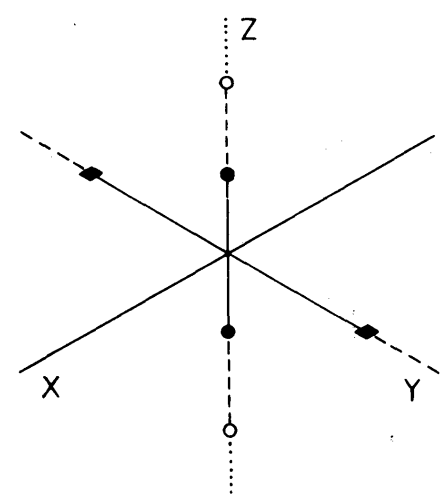

Figure 4. Axial orbits and their stability in a three-dimensional Stäckel potential. The filled squares and the filled and open circles denote the foci. A dashed line indicates orbits that are unstable in one direction; a dotted line indicates the $z$-axis orbits that are unstable in two perpendicular directions.

bifurcation points on the $y$-axis, and to derive $\gamma-\beta$ from the location of the similar points on the $z$-axis. The second pair of bifurcation points on the $z$-axis is less important since it marks the onset of the second instability of the $z$-axis oscillation and the existence of the unstable elliptic closed orbits in the $(x, z)$-plane (de Zeeuw 1985a).

In all of this section we have assumed that $V_{\mathrm{S}}$ is smooth. This requires that $\zeta^{*}(\lambda)$ and $\eta^{*}(\mu)$ and their derivatives are equal at $\lambda=\mu=-\alpha$, and similarly for $\eta^{*}(\mu)$ and $\theta^{*}(v)$ at $\mu=v=-\beta$ (Lynden-Bell 1962). It is in most cases no loss of generality to take $\zeta^{*}(\lambda)=F(\lambda), \eta^{*}(\mu)=F(\mu)$ and $\theta^{*}(v)=F(v)$, so that $\zeta^{*}, \eta^{*}$ and $\theta^{*}$ are the same function $F(\tau)$ - assumed to be smooth - but on adjacent intervals $(\tau=\lambda, \mu$ or $v)$. If one is trying to find the adelphic integrals for an orbit that does not come close to the points where $\lambda=\mu=-\alpha$ and $\mu=\nu=-\beta$, there is no need to require that $V_{\mathrm{S}}$ is smooth there. However, for a global fit to a potential $V$, for example that of a numerical model, this constraint on $\zeta^{*}, \eta^{*}$ and $\theta^{*}$ should be kept in mind.

Tests and applications of the global fitting procedure are being pursued. For a preliminary account, see Wilkinson (1983).

\section{Concluding remarks}

We have shown that near an equilibrium point a general potential may be fitted quite accurately with one of Stäckel form. This is true in particular for potentials with a number of reflection symmetries. One can fit the expansion of a potential $V=V\left(x^{2}, y^{2}, z^{2}\right)$ to quartic order provided that one constraint on the quartic terms is satisfied. This is the case for all potentials of stratified ellipsoids, and many others. Furthermore, one can write the expansions for the integrals of motion in the separable potential that fits $V$ directly in terms of the expansion coefficients of $V$ itself, without any reference to the ellipsoidal coordinates or the best-fitting Stäckel potential. Thus, local fitting produces three integrals of motion immediately. The nature of these integrals was elucidated in Section 2. By a detailed comparison of the results obtained for this case by local fitting and by the (rigorous) averaging method, it is possible to delineate the circumstances under which local fitting is applicable, and also to estimate its accuracy. We do this in the companion paper (de Zeeuw 1985b).

In a description of motion near an equilibrium point as perturbed harmonic motion one assumes that the terms in the expansion of $V$ that are higher than quadratic are small. The results are therefore valid only close to the equilibrium point. When fitting with a Stäckel potential one changes the higher order terms in $V$ in such a way that the expansion converges to a separable one. The examples of Section 4 show that, although the series expansions have a finite radius of 
convergence, in some cases the potential that is fitted is (close to) separable at all radii. The case of the perfect ellipsoid (Section 4.1) shows that the potentials of elliptical galaxies are quite well approximated by potentials of Stäckel form, not only locally, but also globally. The method presented in Section 5 provides a best global fitting Stäckel potential. Perturbation theory, e.g. with slow and fast variables (Lynden-Bell 1973; Contopoulos 1975), applied to the (unperturbed) motion in this Stäckel potential should give an accurate description of motion at all radii.

It is to be expected, however, that in many cases the separable potentials will not give a good global fit, especially if the potential that is fitted has few reflection symmetries. One may then ask whether a particular orbit can be well fitted by an orbit in a potential with exact integrals. We have not addressed this problem.

It should be noted that in non-separable potentials algebraic integrals may arise which exist for one energy only and have a non-quadratic velocity dependence. When considering only one orbit it may therefore be necessary to go beyond fitting with Stäckel potentials (e.g. Hall 1983). It is evident that there remain many challenging problems in the general area of finding approximate analytic integrals for non-integrable systems.

Finally we remark that some special potentials are known which have further integrals. For an account of them in two dimensions see Fris et al. (1965). When rotation is present much less is known although the harmonic case has been well discussed by Freeman (1966) and Hunter (1974), and Vandervoort (1979) has a surprising exact integral for a special potential.

\section{Acknowledgments}

This work owes much to the stimulation of others, especially C. A. Norman, H. C. van de Hulst, M. Schwarzschild and J. J. Binney. Comments by H. C. van de Hulst, F. Verhulst, and the referee, Dr I. C. Percival considerably improved the presentation of the paper. TdZ gratefully acknowledges the hospitality of the Institute of Astronomy in Cambridge, where this work was started. The final part of this work was partially supported by NSF Grants PHY-82-17352 and PHY-84-40263.

\section{References}

Bertrand, J., 1852. J. Math., 17, 121.

Binney, J. J., 1978. Comments Astrophys., 8, 27.

Bogoliubov, N. N. \& Mitropolsky, Y. A., 1961. Asymptotic Methods in the Theory of Nonlinear Oscillations, Gordon and Breach, New York.

Chandrasekhar, S., 1969. Ellipsoidal Figures of Equilibrium, Ch. 3, Yale University Press, New Haven.

Clark, G. L., 1936. Mon. Not. R. astr. Soc., 97, 182.

Contopoulos, G., 1960. Z. Astrophys., 49, 273.

Contopoulos, G., 1963. Astr. J., 68, 763.

Contopoulos, G., 1975. Astrophys. J., 201, 566.

Dall'Acqua, F. A., 1908. Math. Ann., 66, 394.

de Zeeuw, P. T., 1985a. Mon. Not. R. astr. Soc., in press.

de Zeeuw, P. T., 1985b. Mon. Not. R. astr. Soc., 215, 729.

de Zeeuw, P. T. \& Merritt, D. R., 1983. Astrophys. J., 267, 571.

Eddington, A. S., 1915. Mon. Not. R. astr. Soc., 76, 37.

Freeman, K. C., 1966. Mon. Not. R. astr. Soc., 134, 1.

Fris, J., Mandrosov, V., Smorodinsky, Ya. A., Uhlir, M. \& Winternitz, P., 1965. Phys. Lett., $16,354$.

Hagihara, Y., 1974. Cel. Mech., Vol. 2, MIT Press, Cambridge.

Hall, L. S., 1983. Physica D, 8, 90.

Heiligman, G. \& Schwarzschild, M., 1979. Astrophys. J., 233, 872.

Hori, G., 1962. Publs astr. Soc. Japan, 14, 353.

\section{(C) Royal Astronomical Society - Provided by the NASA Astrophysics Data System}


Hunter, C., 1974. Mon. Not. R. astr. Soc., 166, 633.

Illingworth, G., 1981. In: The Structure and Evolution of Normal Galaxies, p. 27, eds Fall, S. M. \& Lynden-Bell, D., Cambridge University Press.

Jacobi, C. G. J., 1866. Vorlesungen über Dynamik, given at Königsberg 1842-43, published by A. Clebsch, Reimer, Berlin.

Kuzmin, G. G., 1956. Astr. Zh., 33, 27.

Levi-Civita, T., 1904. Math. Ann., 59, 383.

Liouville, J., 1846. J. Math., 11, 345.

Liouville, J., 1849. J. Math., 14, 257.

Lynden-Bell, D., 1962. Mon. Not. R. astr. Soc., 124, 95.

Lynden-Bell, D., 1973. In: Dynamics of Stellar Systems, Proc. Third Adv. Course of the Swiss Society of Astr. and Astroph., p. 91, eds Martinet, L. \& Mayor, M., Observatoire de Genève, Sauverny.

Manabe, S., 1979. Publs astr. Soc. Japan, 31, 369.

Morse, P. M. \& Feshbach, H., 1953. Methods of Theoretical Physics, Ch. 5, McGraw Hill, New York.

Ollongren, A., 1962. Bull. Astr. Inst. Neth., 16, 241.

Schwarzschild, M., 1979. Astrophys. J., 232, 236.

Schwarzschild, M., 1981. In: The Structure and Evolution of Normal Galaxies, p. 43, eds Fall, S. M. \& Lynden-Bell, D., Cambridge University Press.

Stäckel, P., 1890. Math. Ann., 35, 91.

Stäckel, P., 1891. Über die Integration der Hamilton-Jacobischen Differential Gleichung mittelst Separation der. Variabeln, Habilitationsschrift, Halle.

Stäckel, P., 1893. Math. Ann., 42, 537.

van de Hulst, H. C., 1962. Bull. Astr. Inst. Neth., 16, 235.

Vandervoort, P. O., 1979. Astrophys. J., 232, 91.

Verhulst, F., 1979. Phil. Trans. R. Soc. London, 290, 435.

Verhulst, F., 1983. In: Asymptotic Analysis II, Lecture Notes in Mathematics, Vol. 985, p. 137, ed. Verhulst, F., Springer-Verlag, Berlin.

Wayman, P. A., 1959. Mon. Not. R. astr. Soc., 119, 34.

Weinacht, J., 1924. Math. Ann., 91, 279.

Whittaker, E. T., 1917. Analytical Dynamics, Cambridge University press.

Whittaker, E. T. \& Watson, G. N., 1902. A Course in Modern Analysis, Cambridge University Press.

Wilkinson, A., 1983. In: Formation and Evolution of Galaxies and Large Structures in the Universe, p. 314, eds Audouze, J. \& Tran Thanh Van, J., Reidel, Dordrecht, Holland. 